\title{
Comparative Analysis of Traffic Congestion Prediction Models for Cellular Mobile Macrocells
}

\author{
Aliyu Ozovehe, Okpo U. Okereke, Anene E. Chibuzo, and Abraham U. Usman
}

\begin{abstract}
Traffic congestion prediction is a non-linear process that involves obtaining valuable information from a set of traffic data and linear models cannot be applied because of the dynamics of combined voice and data traffic on one radio channel of GSM/GPRS access network. However, non-linear problems can easily be modeled using Artificial Intelligent (AI) techniques such as Artificial Neural Networks (ANN) and Adaptive Neuro-Fuzzy Inference Systems (ANFIS). In this work, three types of ANN and an ANFIS models are trained based on busy hour (BH) traffic measurement data taken from some GSM/GPRS sites in Abuja. The models were then used to predict traffic congestion for some macrocells and their accuracy are compared using four statistical indices. It was observed that Group Method of Data Handling (GMDH) model which is one of the ANN models has the best fit and predict better than ANFIS and the other two ANN models. The GMDH model is found to offer improved prediction results in terms of increasing the $R^{2}$ by $20 \%$ and reducing $R M S E$ by $60 \%$ over ANFIS, the closest model to the GMDH in term of prediction accuracy.
\end{abstract}

Index Terms-Artificial Intelligent Network; Quality of Service; Busy Hour Traffic and Traffic Congestion.

\section{INTRODUCTION}

The GSM service providers in Nigeria have not been able to satisfy Quality of Service (QoS) as a result of network congestion [1] and there is dire need for improved QoS in mobile communication services by providing useful tools for predicting $\mathrm{BH}$ traffic congestion which is essential for proper network planning and optimization.

The traffic prediction modeling is a special case of the Box-Jenkins' auto-regressive integrated moving average (ARIMA) models in their framework. However, it is difficult to predict mixed traffic in GSM/GPRS network with ARIMA models because of the bursty nature of the traffic sources and the effects of high-speed channels that characterized the network. Experimental results have shown that the artificial intelligence (AI) techniques are superior to traditional regression or auto-regression techniques as tools for traffic prediction.

This work explored Multiple Layer Perceptron Neural Network (MLPNN), Radial Basis Function Neural Network (RBFNN), Group Method of Data Handling Polynomial Neural Network (GMDH-PNN) and ANFIS models that have found application in traffic prediction [2] - [7]. The aim is to train the computational intelligent techniques for

Published on June 30, 2018

A. Ozovehe, O. U. Okereke, and A. E. Chibuzo are with the Department of Electrical and Electronics Engineering, Abubakar Tafawa Balewa University, Bauchi, Nigeria

A. U. Usman is with the Department of Electrical and Electronics Engineering, Federal University of Technology, Minna, Nigeria

Correspondence: aliyu123oz@gmail.com, +2348030547650 planning and optimization of GSMGPRS macrocell using two years' traffic measurement data as a case study. Performances of the models were determined and recommendations made for their applicability in the study area.

\section{LITERATURE REVIEW}

Basically, ANNs are mostly used for modeling nonlinear statistical data and they mimic the working of neural networks of human brains. Fig. 1 shows a simple neuron model and (1) relates the input to the output of the model.

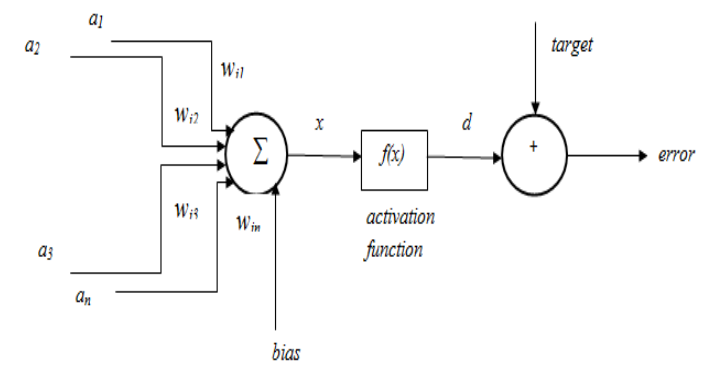

Fig. 1. A common model for neuron

It is obvious from Fig. 1 that the neuron is a processing element that takes a number of inputs, $a_{1}, a_{2} \ldots a_{n}$, weight them with

weights, $w_{1}, w_{2} \ldots w_{n}$, sums them up together with a bias parameter, bias, to get $x$. $x$ is then processed by the activation function, $f(u)$ and the neuron output error, $e$, is calculated by subtracting the output, $d$, from the target value, $t$, as in (1):

$$
e=t-d
$$

The essence of neuron model is to minimize the output error, $e$, according to some optimization criteria to improve goodness of fit.

All the neurons in NNs are trained using a block of inputs and outputs set of data in order for the NNs to have knowledge about the problem. The training can be classified into two, supervised and un-supervised training. The former needs pre-defined set of training data that reflect the network behaviour. The network target is already known and it is compared to the output when the input is applied to the network. The learning rule modifies $w_{1}, w_{2} . w_{n}$, and bias so that the outputs are closer to the target [8]. 
However, $w_{1}, w_{2} \cdot w_{n}$, and bias parameters are updated solely with inputs data in unsupervised training.

\section{A. Review Stage Multilayer Perceptron Neural Network (MLPNN)}

MLPNN is an extension of the neuron model and connections can only be unidirectional in the network. Neurons in proceeding layer can only be linked to neurons in the successive layer and the no loops in MLPNN architecture means computation can take place uniformly from input neurons to output neurons [9]. MLPNN has three layers of neurons in its simplest form as shown in Fig. 2 and uses sigmoid activation function in hidden and output layers for supervised learning algorithm that calculate the change in network weights commonly referred to as backpropagation.

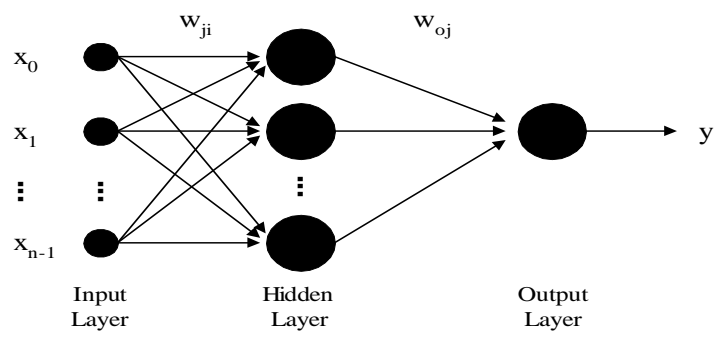

Fig. 2. Simple MLPNN architecture

Thus, MLP network output can be expressed as in (2).

$y=\frac{\sum_{j=0}^{M}\left(w_{0 j}\left(F_{h}\left(\sum_{i=0}^{N} w_{j i} x_{i}\right)\right)\right)}{F_{0}^{-1}}$

The training process minimizes the mean square error (MSE) by regulating the $w_{1}, w_{2} \ldots w_{n}$ using a block set of data as given in (3).

$$
M S E=\frac{1}{N} \sum_{i=1}^{N}\left(y_{i}-\hat{y}_{i}\right)^{2}
$$

where $y_{i}$ is the measured output value and $\hat{y}_{i}$ is the predicted output of the network, while $N$ is the sampling numbers.

MLPNN of many layers can easily be designed, [10] has shown that only one hidden layer is necessary to get good result from the network if sigmoid activation function is used.

\section{B. Radial Basis Function Neural Network (RBFNN)}

RBFNN is an MLPNN network that uses radial basis function as an activation function in the hidden layer. Fig. 3 shows the generalized RBFNN.

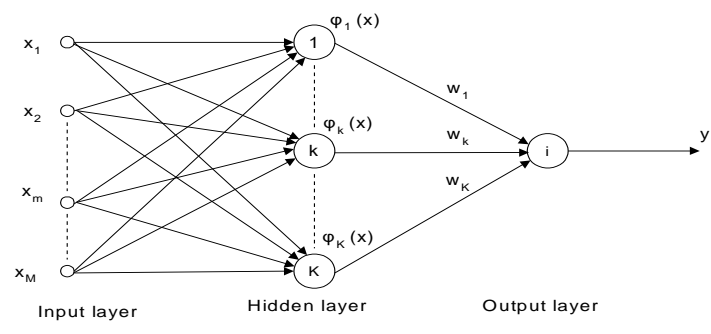

Fig. 3. Simple RBFNN configuration

Input into RBFNN is usually modeled as given by (4):

$x \in R^{n}$

where $R$ is a real numbers.

The RBFNN output as a non-vector function can be expressed as in (5):

$$
\varphi(x)=\sum_{i=1}^{N} a_{i} \rho\left(\left\|x-c_{i}\right\|\right)
$$

RBFNN depends exclusively on length of interval between centre vectors $c_{i}$ and its norm is taken as Euclidean distance which becomes Gaussian basis function when

$$
\lim _{\|x\| \rightarrow \infty} \rho\left(\left\|x-c_{i}\right\|\right)=0
$$

Use is made of $a_{i}, c_{i}$ and $\rho$ parameters for optimal fit between RBFNN output and the data.

RBFNN networks are trained by properly chosen the centre vectors $c_{i}$ and then use $w_{i}$ to fits a linear model with respect to some objective (least squares) function. Back propagation step may be performed for optimal $a_{i}, c_{i}$ and $\rho$ parameters.

\section{Group Method of Data Handling Polynomial Neural Network (GMDH-PNN) Model}

GMDH-PNN was developed as a model for obtaining high order input- output relationship in time-series problems by identifying non-linear relationships between inputs and outputs data [11]. GMDH is an inductive unidirectional polynomial neural network that is made of large number of layers and each layer contains many neurons. All neurons in GMDH shell have two inputs and one output as shown in Fig. 4.

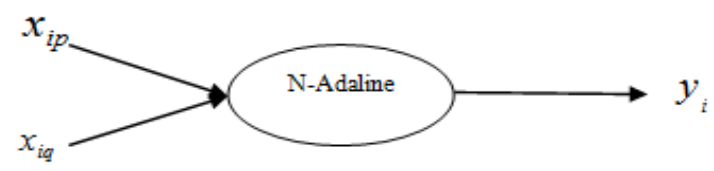

Fig. 4. A Single GMDH Neuron Model

GMDH neuron model can be expressed mathematically as a second order polynomial network (PNN) model and the most popular function used in GMDH is the KolmogorovGabor polynomial with base function as in (6). 
$y_{i}=f\left(x_{i p}, x_{i q}\right)=a_{0}+a_{1} x_{i p}+a_{2} x_{i q}+a_{3} x_{i p} x_{i q}+a_{4} x_{i p}^{2}+a_{5} x_{i q}^{2}$

The function $f$ is equipped with six $x$ factor estimating $\left\{\left(x_{i p}, x_{i q}\right), i=1,2,3, \ldots N\right\}$ system and optimal output of $\left\{\left(y_{i}\right), i=1,2,3, \ldots N\right\}$ for all dependent two-variable samples [12].

Each term of Kolmogorov-Gabor polynomial contributes differently and the GMDH network removes the terms that do not contribute significantly layer by layer using selforganizing arithmetic. The goal of modeling can be reached if the function $f$ is planned according to minimum squares error as shown as in (7).

$$
\operatorname{Min} \sum_{k=1}^{N}\left[\left(f\left(x_{i p}, x_{i q}\right)-y_{i}\right)^{2}\right]
$$

Application of ANNs to traffic prediction by [1] - [2], [7] and [13] - [18] showed that MLPNN, RBFNN and GMDHPNN can predict traffic with very high accuracy.

\section{Adaptive Neuro-Fuzzy Inference Systems (ANFIS)}

ANFIS utilizes the learning ability, adaptability and knowledge discovery of neural networks and fuzzy systems to represent knowledge and figure out inaccurate information [19]. The ANFIS used in this work is a first order Takagi-Sugeno-Kang (TSK) that has inputs, $x$ and $y$ and an output $f(x, y)$ as shown in Fig. 5 .

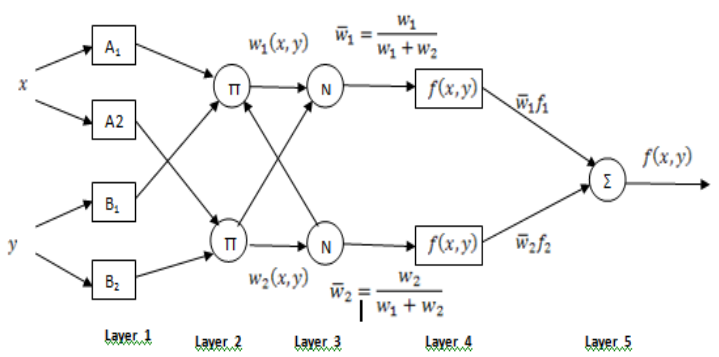

Fig. 5. Simple Configuration of ANFIS

The logic behind the working of TSK inference system can be stated as:

If $x$ is $A_{i}$ and $y$ is $B_{i}$,

THEN fi $=\mathrm{p}_{\mathrm{i}} x+\mathrm{q}_{\mathrm{i}} y+\mathrm{r}_{i}$

$A_{i}$ and $B_{i}$ represents linguistic labels for the input $x$ and $y$ respectively and $f_{i}$ stands for adaptive linear function of the system.

Layer 1 inputs are fuzzified in this layer by using the membership functions whose parameters are adaptable. The parameters of the MFs are known as antecedent parameters. Fig. 6 shows the most common membership function which is basically a Gaussian bell function.

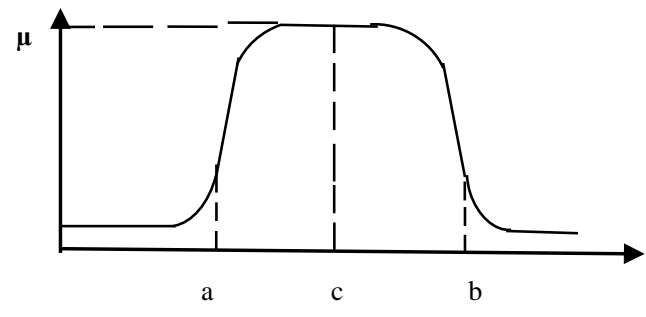

Fig. 6. Bell membership function

The bell function is expressed as in (8):

$\mu_{y i}(\hat{y})=\frac{1}{1+\left[\frac{x-c_{i}}{a_{i}}\right]^{2 b_{i}}}$

where $\mathrm{a}, \mathrm{b}$ and $\mathrm{c}$ are antecedent parameters.

Layer 2 is a hidden layer with fixed nodes that perform the rule of firing strength as given in (9).

$w_{i}^{n}=\prod_{i=1}^{n} \mu_{A i}\left(x_{i}\right) \mu_{B i}\left(y_{i}\right)$

Layer 3 this layer produces an output called normalized firing strength of each rule according to (10).

$$
\overline{w_{i}}=\frac{w_{i}}{\sum_{j=1}^{2} w_{j}}
$$

Layer 4 is an adaptive node where every node is a linear function of the inputs with some adaptive gains and constant parameters. Each node has a function given in (11).

$\overline{w_{i}} f_{i}=\overline{w_{i}}\left(p_{i} x+q_{i} y+r_{i}\right)$

where, $p_{i}, q_{i}$ and $r_{i}$ are referred to as consequent parameters and use is made of least-squares (LS) method in practice to get their optimal values.

Layer 5 defuzzification is performed in this layer to generate a crisp output for the systems as expressed in (12).

$$
f(x, y)=\sum_{i} \overline{w_{i}} f_{i}=\frac{\sum_{i} w_{i} f_{i}}{\sum_{i} w_{i}}
$$

note that $\overline{w_{i}} f_{i}$, is layer 4 output.

To estimate the parameters of membership function, ANFIS is trained using either back propagation or least squares estimation and back-propagation. 


\section{Methodology}

The experimental setup for collecting and processing the traffic data used in this work is shown in Fig. 7. The setup comprises of base station subsystem (BSS) and network subsystem (NSS) connected to standalone system called network management system (NMS) [1].

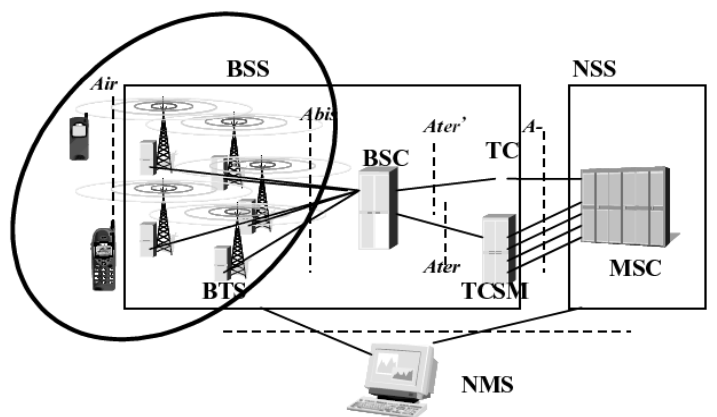

Fig. 7. System for Collecting Data

NMS is the functional entity from which the service provider monitors and controls the entire network. The data used in this work was extracted from the NMS with the help of Ericsson Business intelligent (BI) tools installed on the standalone computer and exported to Microsoft Excel environment and part of the processed data for CELL01 is shown in Table I.

\begin{tabular}{ccc}
\multicolumn{3}{c}{ TABLE I: TRAFFIC DATA FOR CELL01 } \\
\hline \hline \multicolumn{2}{c}{ CELL01 DATA } & \\
CSSR & TRAFFIC & TCH CONG \\
\hline 57.92 & 29.88 & 2.67 \\
56.9 & 30.73 & 2.98 \\
58.71 & 23.73 & 3.01 \\
42.35 & 17.75 & 3.9 \\
46 & 19.74 & 3.97 \\
46.34 & 17.54 & 5.12 \\
47.26 & 19.31 & 7.73 \\
48.62 & 19.75 & 8.84 \\
$\ldots$ & $\ldots$ & $\ldots$ \\
$\ldots$ & $\ldots$ & $\ldots$ \\
65.8 & 18.87 & 32.1 \\
\hline \hline
\end{tabular}

To develop a good prediction model for the congestion, the selection of the input variables must be closely associated with the TCH congestion values and there must be a strong linear correlation between the traffic parameters (CSSR, HOSR, DCR, SDCCH congestion and busy hour $\mathrm{BH}$ traffic) and $\mathrm{TCH}$ congestion. Correlation test showed that traffic channel $(\mathrm{TCH})$ congestion depend only on call setup success rate (CSSR) and BH traffic at cell level. An average correlation coefficient value of 0.9 was observed between TCH congestion and CSSR while 0.6 was observed between $\mathrm{TCH}$ congestion and $\mathrm{BH}$ traffic [1].

To fit $\mathrm{BH}$ traffic versus $\mathrm{TCH}$ congestion pairs for each Cell:

- newff function of MATLAB was used to create an MLPNN 2-3-1 (two inputs, three hidden layer and one input). MATLAB rand was used to set a random seed ('seed', 447944968) to avoid randomness experienced in each run of the MATLAB program. The network was trained with LMA in order to avoid over fitting and early stopping.

- An optimal RBFNN performance was achieved when 0.5 spread constant is used as default data length to fit BH traffic/CSSR versus TCH congestion pairs for each Cell.

- $\quad$ For standard GMDH-PNN model, BH traffic/CSSR and TCH congestion pairs of each cell was fit into GDMH shell which create a second order polynomial network. The traffic data was imported in to GMDH shell environment in XLS format to train and validate the model for predicting the busy hour congestion of the cell using k-fold cross-validation to split the whole dataset into ratio 40:60 for training and testing respectively.

- For optimal performance of ANFIS model 25 was used as number of fuzzy MF per input, Bell membership function (MF) was specified as the type of fuzzy MF and 300 was taken the number of epochs [20] before the training.

Table II shows the various parameters used for the four models.

TABLE II: PARAMETERS OF THE MODELS

\begin{tabular}{ll}
\hline \hline Parameter & Value \\
\hline No. of MLP Hidden Layer 1 & 1 \\
No. of MLP Hidden Layer 2 & 3 \\
No. of epochs & 300 \\
No. of RBF neurons & Default length of data \\
RBF's spread & 0.5 \\
RBF's error goal & 0.01 \\
GMDH's training & $\mathrm{k}-1$ \\
No. of ANFIS MF & 25 \\
\hline \hline
\end{tabular}

Four standard statistical performance evaluation criteriamean absolute error (MAE), standard deviation (SD) and root mean square error (RMSE) and R-Square $\left(\mathrm{R}^{2}\right)$ values as in $(13-16)$ were used to measure the performances of these models.

$\mathrm{M} A E=\frac{1}{N} \sum_{i=1}^{N} E_{i}$

where $E_{i}=\left|y_{i}-\hat{y}_{i}\right|$ and $N$ is the sampling numbers.

$M A E$ measures how close the predictions are to the measured congestion.

$S D=\sqrt{\frac{1}{N-1}\left(\sum_{i=1}^{N} E_{i}^{2}-N \cdot \mu^{2}\right)}$

SD quantifies the total deviation in a set of samples data and a low value depicts that the samples data are close to the mean of the set.

$R M S E=\sqrt{\frac{\sum_{i=1}^{N}\left(y_{i}-\hat{y}_{i}\right)^{2}}{N}}$ 
RMSE aggregates the amount of errors in predictions and the lower the value the higher the accuracy of the prediction.

$$
R^{2}=1-\frac{\sum_{i=1}^{N}\left(y_{i}-\hat{y}_{i}\right)^{2}}{\sum_{i=1}^{N}\left(y_{i}-\bar{y}_{i}\right)^{2}}
$$

where, $\bar{y}_{i}$ is input data (congestion) mean

$R^{2}$ shows the closeness of the predicted values to the fitted regression line.

\section{RESULT AND DISCUSSION}

The traffic measurement data was split into parts: $40 \%$ training and $60 \%$ testing for the four models. Plots for the training errors, validation errors and errors test performance of the four CELLs are shown in Fig 8 - 11 while Tables IIIIV present the numerical value of $\mu, \sigma, \mathrm{RMSE}$ and $\mathrm{R}^{2}$ for the four CELLs and the models.

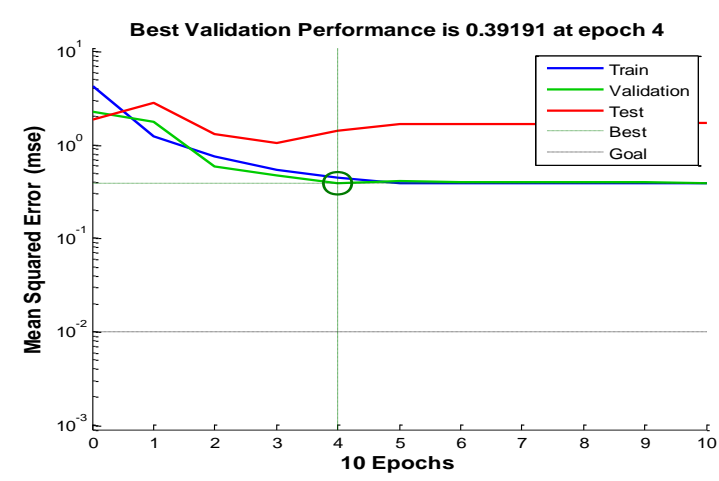

Fig. 8: CELL1 training, validation and errors test

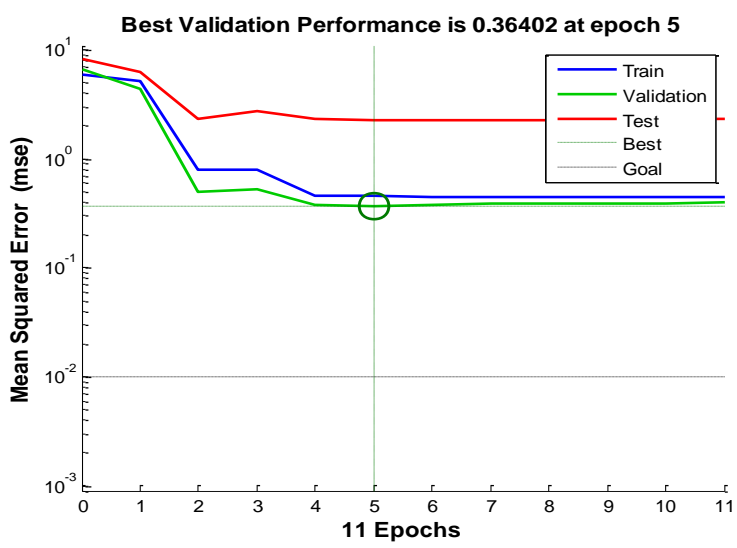

Fig. 9: CELL2 training, validation and test errors test

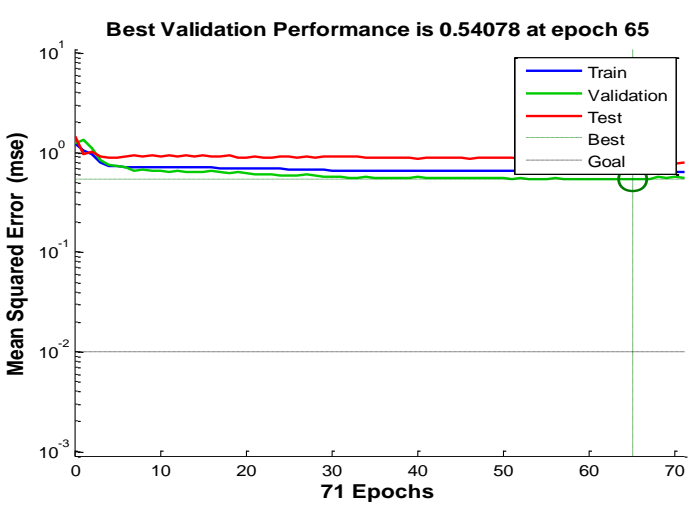

Fig. 10. CELL3 training, validation and errors test

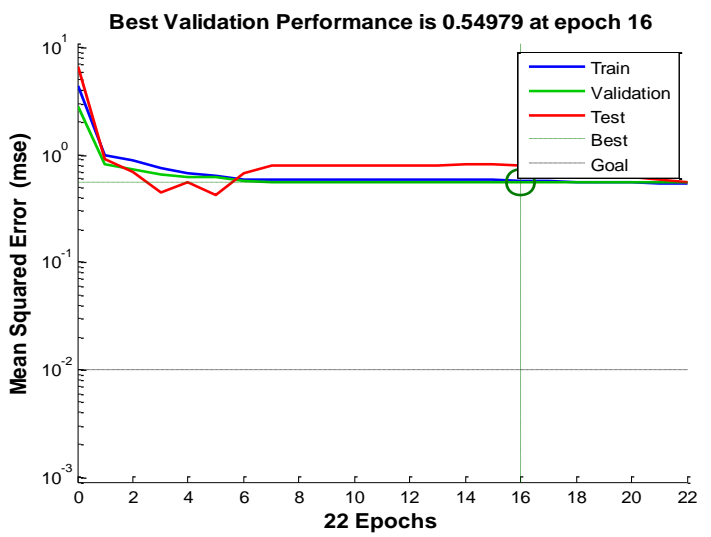

Fig. 11. CELL4 training, validation and errors test

TABLE III: MAE PERFORMANCE OF THE MODELS

\begin{tabular}{ccccc}
\hline \hline Site & MLP & RBF & ANFIS & GDMH \\
\hline CELL01 & 13.53 & 6.79 & 4.22 & 2.65 \\
CELL02 & 7.12 & 0.33 & 4.65 & 3.83 \\
CELL03 & 18.42 & 0.39 & 7.32 & 0.67 \\
CELL04 & 10.75 & 1.68 & 3.91 & 2.13 \\
Average & 13.15 & 2.3 & 5.02 & 2.32 \\
\hline \hline
\end{tabular}

TABLE IV: SD PERFORMANCE OF THE MODELS

\begin{tabular}{ccccc}
\hline \hline Site & MLP & RBF & ANFIS & GDMH \\
\hline CELL 1 & 12 & 12.43 & 5.69 & 3.91 \\
CELL 2 & 7.19 & 0.26 & 5.3 & 4.56 \\
CELL 3 & 14.29 & 0.22 & 8.81 & 0.94 \\
CELL 4 & 8.16 & 1.38 & 5.07 & 4.72 \\
Average & 11.52 & 3.52 & 6.22 & 3.53 \\
\hline \hline
\end{tabular}

TABLE V: RMSE PERFORMANCE OF THE MODELS

\begin{tabular}{ccccc}
\hline \hline Site & MLP & RBF & ANFIS & GDMH \\
\hline CELL 1 & 17.96 & 13.36 & 7.05 & 3.91 \\
CELL 2 & 10.09 & 0.41 & 7.04 & 4.62 \\
CELL 3 & 23.28 & 0.44 & 11.44 & 0.94 \\
CELL 4 & 13.47 & 2.11 & 6.39 & $4 . .77$ \\
Average & 17.46 & 4.08 & 7.98 & 3.16 \\
\hline \hline
\end{tabular}

TABLE VI: $R^{2}$ PERFORMANCE OF THE MODELS

\begin{tabular}{ccccc}
\hline \hline Site & MLP & RBF & ANFIS & GDMH \\
\hline CELL 1 & 0.34 & -2.62 & 0.92 & 1 \\
CELL 2 & 0.44 & 0.13 & 0.7 & 0.97 \\
CELL 3 & 0.14 & 0.44 & 0.79 & 1 \\
CELL 4 & 0.36 & -0.04 & 0.87 & 1 \\
Average & 0.32 & -0.61 & 0.82 & 0.99 \\
\hline \hline
\end{tabular}


Fig. 12 - 13 showed that the analysis of the models performance using MAE, SD, RMSE and $R^{2}$.

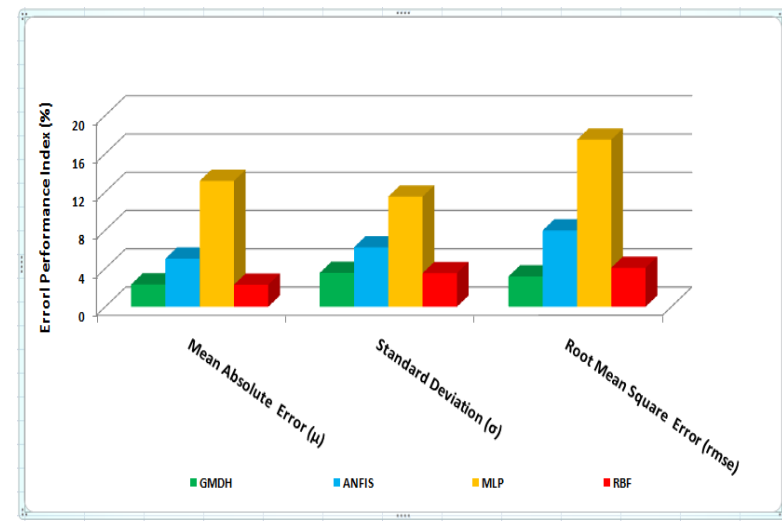

Fig. 12. Average error performance of the four models

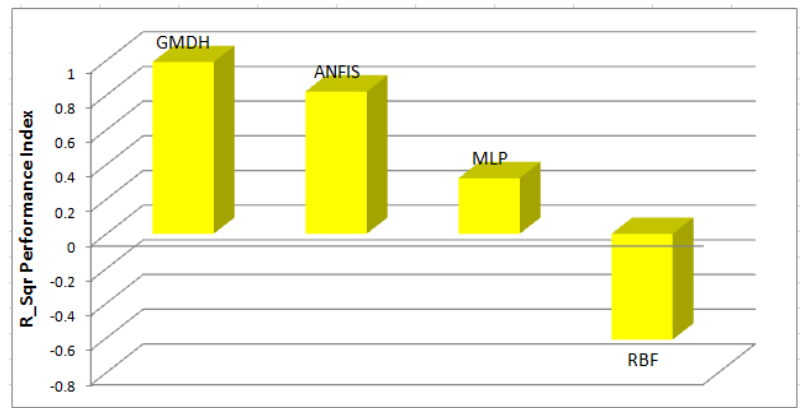

Fig. 13. Average goodness performance of the four models

Fig. 13 showed that GMDH-PNN model has the best performance using the most important statistical measure, $R^{2}$. The average $R^{2}$ performance of the GMDH-PNN, ANFIS, MLPNN and RBFNN models are 0.99, 0.82, 0.32 and -0.61 respectively.

Using MAE, SD and RMSE in Fig. 12, the RBFNN model performances compete favorably with GMDH-PNN model. However, RBFNN negative value $R^{2}$ makes it an inappropriate model for this prediction when compared to other models.

The RMSPE values of MLPNN model is extremely too high in all the Cells when compared with other Models and hence is not good for modeling the data. Also, the ANFIS model performance is next to GMDH-PNN model in term of predictions accuracy while the performance RBFNN model is the worst.

\section{CONCLUSION}

GMDH-PNN predicted TCH congestion better than any other models because it produces lower error in terms of $M A E, S D, R M S E$ and higher $R^{2}$ between actual and predicted TCH congestion. These suggest the suitability of GMDH-NN for prediction of TCH congestion for effective management of macrocell network resources in cellular mobile.
[2] S. Ardhan, S. Satsri, V. Chutchavong and O. Sangaroon, "Improved model for traffic fluctuation prediction by Neural network," International Conference on Control, Automation and Systems, Seoul, Korea, Oct. 17-20,2007.

[3] F. Z. Mohamed and E. Halima, "Analysis and prediction of real network traffic, Journal of Networks," Vol. 4, No. 9, November 2009.

[4] S. Garba, B.G. Bajoga, M.B. Mu'azu, D.D. Dajab, and U.F AbduAguye, Development of an ANFIS-based QoS model for a GSM service provider (MTN Nigeria Kano region network), 3rd IEEE International Conference on Adaptive Science and Technology (ICAST 2011).

[5] R. E. Sivakumar, K Ashok, and G. Sivaradje, "Prediction of traffic load in wireless network using time series model," Department of Electronics and Communication Engineering Pondicherry Engineering College, Puducherry-605 014, India, 978-1-61284-764$1 / 11 / 2011$.

[6] P. Gaurav, M. S Khadim, and A.K. Choudhary, "Telecom voice traffic prediction for gsm using feed forward neural network," International Journal of Engineering Science and Technology (IJEST), ISSN: 0975-5462 Vol. 5 No.03, March 2013.

[7] E.O Oladeji, E.N Onwuka, M. A. Aibinu, "Determination of voice traffic busy hour and traffic forecasting in global system of mobile communication (GSM) in Nigeria, $11^{\text {th }}$ Malaysia International Conference on Communications, 26th-28th November 2013.

[8] P. Aurabind, R. Anubhav, M. Vaibhav, and G. Anshul, "Development of a smart grid prototype for the proposed $33 \mathrm{kv}$ distribution system," Measurement of Electrical Engineering, National Institute of Technology Rourkela, 2011.

[9] A. Simaneka, "Development of models for short-term load forecasting using Artificial Neural Network," Master's Thesis, Faculty of Engineering, Cape Peninsula University of Technology, November 2008

[10] E. Ostlin, H. J Zepernick, and H. Suzuki, "Macrocell radio wave propagation prediction using an artificial neural network," IEEE Semiannual Vehicular Technology Conference, 1, 57-61, 2004.

[11] B. Koo, S. Lee, W. Kim, and J. H. Park, "Comparative study of shortterm electric load forecasting," Fifth International Conference on Intelligent Systems, " Modeling and Simulation, 2014.

[12] K. Atashkari, N., Nariman-Zadeh, M. Gölcü, A. Khalkhali, and A. Jamali, "Modelling and multi-objective optimization of a variable valve-timing spark-ignition engine using polynomial neural networks and evolutionary algorithms," Energy Conversion and Management Journal, 48(3) 1029-1041, 2007.

[13] S Edmund, Yu. Roger and C.Y. Chen, "Traffic prediction using neural networks, school of computer and information sciences," Syracuse University, Syracuse, New York, 0-7803-0917-0, 1993.

[14] T. Edwards, D.S.W. Tansley, R. J. Frank, N. Davey, "Traffic trends analysis using neural networks," Faculty of Information Sciences University of Hertfordshire Hatfield, Herts., UK, AL10 9AB, 1998.

[15] H. Jason and M. Philip, The limitations of artificial neural networks for traffic prediction, School of Engineering, University of Durham, Durham, UK \{J.L.Hall, Philip.Mars\}@durham.ac.uk., 1998.

[16] E. D. Markus, O. U. Okereke and J. T. Agee, "Predicting Telephone Traffic Congestion using Multi-Layer Feedforward Neural Networks," Advanced Materials Research Vol. 367 pp 191-198, 2012.

[17] M.A Raheem, and O.U. Okereke, "A Neural Network Approach to GSM Traffic Congestion Prediction", American Journal of Engineering Research (AJER) e-ISSN: 2320-0847 p-ISSN: 23200936 Volume-03, Issue-11, pp-131-138, 2014.

[18] A. Ozovehe, "Group Method of Data Handling for Modeling Macrocell Traffic Congestion, "Zaria Journal of Electrical Engineering Technology, Department of Electrical and Computer Engineering, Ahmadu Bello University, Zaria, Nigeria. Vol. 4 No. 1 and 2, March and September, ISSN: 0261-1570, pp. 46-52, 2015.

[19] M. Negnevitsky, Artificial Intelligence, Pearson Education Limited, 2005.

[20] MATLAB, "Matlab software," Neural Network Toolbox ${ }^{\mathrm{TM}}$, 19842013.

\section{REFERENCES}

[1] O. Aliyu, U. O Okpo, E. C. Anene and U. U. Abraham, "Busy hour traffic congestion analysis in mobile macrocells," Nigerian Journal of Technology (NIJOTECH) Vol. 36, No. 4, pp. 1265-1270, October 2017. 


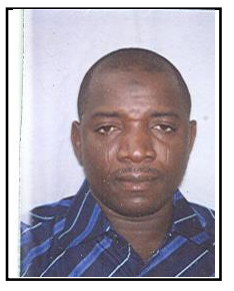

2012.

$\mathrm{He}$ is a Member of Institute of Electrical/Electronics Engineer (2008), Nigerian Society of Engineer (2007), Member Council for Regulation of Engineering in Nigeria (2008), Member IEEE, Nigerian Institute of Electrical/Electronics Engineer (2009). He has just defended PhD degree in Communication Engineering at Electrical/Electronic Engineering Programme of Abubakar Tafawa Balewa University Bauchi, Nigeria. His major fields are Electromagnetic Compatibility, Radio wave Propagation and Teletraffic Engineering.

Born at Okene in Kogi State of Nigeria on May, 15th 1973. He received his degrees of B.Eng (Elect Engineering) and M.Sc (Electrical/Electronics) in 1998 and 2006 from Bayero University Kano Nigeria and University of Lagos Nigeria respectively.

He lectured in Electrical/Electronic Engineering department of Kaduna Polytechnic in 2000/2001, worked with Nigerian Telecommunication Limited (NITEL) 2001 - 2006 and worked on Airtel project (Cellular network provider in Nigeria) from 2006 - 